\title{
IOF World Congress on Osteoporosis \& 10th European Congress on Clinical and Economic Aspects of Osteoporosis and Osteoarthritis
}

\author{
Plenary Lectures Abstracts
}

\section{PL1 - CELLULAR CONTROL OF BONE MASS}

R. Baron ${ }^{1, *} ;{ }^{1}$ Endocrine Unit, MGH, Harvard Medical School, Boston, United States

Abstract: Maintenance of skeletal mass and establishment of peak bone mass are based upon the proper regulation of bone remodeling, which depends on the coordinated actions of boneresorbing osteoclasts and bone-forming osteoblasts. With age, bone formation decreases below the level required to compensate for the often-increased bone resorption, leading to osteoporosis with its associated fractures. It is of clinical importance to understand and integrate the cellular events that take place during bone remodeling and the mechanisms by which they are regulated or altered by disease or by therapeutic intervention.

Activation of the remodeling cycle is initiated by hematopoietic osteoclast precursors and the formation of osteoclasts. This process is under the control of two main cytokines, M-CSF and RANKL. Importantly, both of these cytokines are synthesized and secreted by bone marrow stromal cells and osteoblasts. This establishes the first homeostatic link between bone formation and resorption since the number and activity of osteoclasts is under the control of osteoblasts/stromal cells. At the end of the resorption phase of the remodeling cycle osteoblasts precursors migrate to the recently resorbed site and fill the remodeling space, ultimately replacing the matrix degraded by osteoclasts by a theoretically equivalent amount of newly synthesized matrix. This phenomenon is called "coupling" and significant advances have been recently made in the identification of factors essential for this process, which requires active osteoclasts for the induction of bone formation. Finally, major progress has been made in our understanding of the differentiation and function of osteoblasts and the regulation of bone formation, allowing the design of new anabolics.

These advances in understanding of the cellular basis of bone remodeling have allowed not only the design of novel molecules now ready to reach the market, but also a much better understanding of some of the unwanted effects of these compounds on bone remodeling, bone structure and bone mass.

Anti-resorptives that lead to entirely inactive or absent osteoclasts prevent coupling and lead to markedly decreased bone formation rates whereas molecules that specifically target functional molecules in osteoclasts prevent major decreases in bone forma- tion. Finally, novel anabolics not only increase bone formation but also alter bone resorption through effects on the osteoblastdependent regulation of osteoclasts.

Disclosure of Interest: Consultant / Speaker's bureau / Advisory activities with: Amgen, Merck, Novartis

\section{PL2 - OSTEOPOROSIS, HIP FRACTURES, AND THE BIOL- OGY OF AGING}

S. Cummings ${ }^{1, *} ;{ }^{1}$ San Francisco Coordinating Center, San Francisco, United States

\begin{abstract}
Hip fracture, mortality and many diseases increase exponentially with age. They share the common causal denominator of 'aging' of cells and tissues. There are two basic classes of cellular aging: degeneration of differentiated cells, and loss of ability to regenerate tissues. First, differentiated cells, such as muscle cell, gradually lose their capacity to produce energy (ATP); enzymes, organelles, and DNA accumulate damage, and older cells lose the ability to clear this damage (authophagy). Garbage builds up and cells die. Second, the ability of stem cells to regenerate tissues (such as injured muscle) also decrease with aging at least partly because processes including shortening of telomeres, damages DNA and cells lose the ability to repair the damage. Cells lose the ability to replicate; they become senescent.

These cellular changes lead to a cycle of damage, decreased clearance of damage, decreased renewal of tissues, with accumulation of cellular garbage and progressive loss of function. Such downward spirals lead to exponential rates of failure. Therefore, the downward spiral of cellular aging would lead exponential increases in mortality, disability, and diseases such as hip fracture. Caloric restriction slows almost all of these processes of aging and extends healthy lifespan. Exercise, rapamycin and resveratrol (and other sirtuin stimulants) also appear to have 'anti-aging' properties. Increasing mitochondrial function in muscle may also 'slow aging.' Several of these anti-aging interventions also slow or prevent bone loss. Therefore, slowing aging represents a new, and increasingly imaginable, approach to postponing the risk of hip fracture.
\end{abstract}

Disclosure of Interest: None Declared 


\section{PL3 - WHY DO BONES BREAK: CONTRIBUTIONS OF CORTICAL AND TRABECULAR BONE}

M. Bouxsein ${ }^{1, *} ;{ }^{1}$ Center for Advanced Orthopaedic Studies, Harvard Medical School, Boston, United States

\begin{abstract}
The relative contribution of cortical and trabecular bone to whole bone biomechanical behavior is poorly understood. Yet, this information is fundamental to improved understanding of the origins of skeletal fragility, assessment of fracture risk and efficacy of osteoporosis therapies. Experimental and analytical studies have shown the role of cortical vs. trabecular bone varies with skeletal site (and even within a skeletal site), loading condition, as well as age and disease severity. In the vertebral body, experimental studies using cadaveric vertebrae reported that the cortical shell accounts for 10 to $75 \%$ of vertebral strength. More recent studies using finite element analysis (FEA) extend these observations, reporting that the contribution of the cortical shell is greatest at the mid-vertebral body and lowest at the endplates, and that the cortex contribution increases as trabecular bone volume decreases. At the proximal femur, FEA studies also show heterogeneity in the contribution of cortical vs. trabecular bone by location, with only $30 \%$ of load carried by cortical bone at the subcapital region compared to $80-95 \%$ of load carried by cortical bone at the base of the femoral neck and intertrochanteric region. Finally, at the distal radius, $\mu \mathrm{FEA}$ data indicate that the proportion of load carried by cortical bone also varies with location, with approximately $60 \%$ of the load carried by cortical bone at the ultradistal radius. Altogether these studies indicate a key, and perhaps previously underappreciated, contribution of cortical bone to whole bone strength at all skeletal sites. This presentation will critically review the recent results on load sharing between trabecular and cortical bone, and discuss the implications with regard to fracture risk prediction and mechanisms underlying skeletal fragility and anti-fracture effects of therapeutic interventions.
\end{abstract}

\section{Disclosure of Interest: None Declared}

\section{PL4 - DO MEN AND WOMEN FRACTURE AT THE SAME BONE MINERAL DENSITY?}

J. A. Kanis ${ }^{1, *} ;{ }^{1}$ WHO Collaborating Centre for Metabolic Bone Diseases, University of Sheffield Medical School, Sheffield, United Kingdom

\begin{abstract}
In order to test the hypothesis framed in the title, it is necessary to consider several elements of risk. The first relates to the gradient of fracture risk i.e. the increase in fracture risk per $\mathrm{SD}$ decrease in BMD. For example, the risk of fracture increases approximately 1.6-fold for each SD decrement in the measurement. Thus, an individual with a measurement of 3 standard deviations below the average value for age would have a $1.6^{3}$ or greater than 4 -fold higher risk than an individual with an average BMD. A second consideration concerns the absolute risk and whether this differs between men and women. Many studies that have examined fracture risk in men and women have come to disparate conclusions concerning the relationship between fracture risk and BMD. There are several reasons for these discrepancies: Firstly, the relation between BMD and fracture risk changes
\end{abstract}

with age, so that age-adjustment is required. Second, a difference between sexes in the gradient of risk (relative risk per SD decrease in BMD) could be the result of differences in the SD of measurements. Third, data derived from referral populations of osteoporotic men and women are likely to be biased. Thus these questions are best addressed by the prospective study of population based cohorts that include both sexes in the same sampling frame. The few studies available show that the risk of hip fracture is similar in men and women for any given absolute value for BMD measured at the hip, as is the gradient of risk. Likewise, the risk of vertebral fracture is also similar in men and women for any given lumbar BMD. Whether fracture risks differ at different sites of measurement and for different fracture outcomes is not known. By contrast, the long term probability of fracture is higher in women than in men since probability depends also on the risk of death and women live longer than men. The similarity in fracture risk between men and women of the same age and for any given BMD is likely to be a favourable artefact of BMD testing since the test does not measure true bone density and is influenced by skeletal size. A side effect of the artefact is that a similar cut-off value for hip BMD that is used in women can be used in the diagnosis of osteoporosis in men - namely, a value for BMD $2.5 \mathrm{SD}$ or more below the average for young adult women.

\section{Disclosure of Interest: None Declared}

\section{PL5 - REGION SPECIFIC FRACTURE RISK AND RESPONSE TO THERAPY}

E. Seeman ${ }^{1, *} ;{ }^{1}$ Austin Health, University of Melbourne, Melbourne, Australia

Abstract: Four abnormalities in modelling and remodelling and the structural decay they produce are rational targets for risk assessment, treatment allocation and monitoring. (i) Reduced bone formation and (ii) continued resorption by the basic multicellular unit (BMU) produce a negative BMU balance so each remodelling cycle decays bone, more so after menopause when (iii) remodelling intensity increases and resorption depth transiently worsens. Cavities concentrate stress predisposing to microcracks and remove trabeculae reducing vertebral and metaphyseal compressive strength. Endocortical and intracortical remodelling cause cortical porosity and thinning, relatively more so at sites with thin cortices (vertebrae, metaphyses, superior femoral neck). Cortical surface/volume increases as resorption increases the intra- and endocortical surface exposing a decreasing cortical volume to more remodelling; remodelling in cortex adjacent to marrow, the main site of bone loss after 65 yrs, leaves chaotically connected cortical remnants measured as 'trabecular' bone underestimating true loss of trabecular bone and strength. Porosity calculated from residual compact-appearing cortex omits porosity produced by remodelling of the inner cortex; this underestimates the increase in porosity and nonvertebral fracture risk. This decay is not offset because (iv) periosteal apposition is minimal. Thus, assessing risk is not based on quantifying structural decay and its cellular basis.

Antifracture efficacy depends on targeting the 'big four' and the structural decay; no surface, no structure to preserve or build 
upon. Antiresorptives reduce remodelling and perhaps the negative BMU balance. Partial filling of existing cavities and suppression of new BMUs partly reverses fragility, reduces progression of porosity, cortical and trabecular thinning but cannot replace trabeculae or reverse severe porosity. PTH increases remodelling and may produce a positive BMU balance depositing bone on trabecular and endocortical surfaces and perhaps the periosteum but intracortical porosity may transiently increase. Assessing treatment - why, who, when, what drug, how long, success or failure, is not based on quantifying structural decay, its cellular basis or changes during treatment in each. Patients are treated similarly despite the heterogeneous structural and cellular basis of fragility. Fracture is structural failure; risk assessment, choice and monitoring of therapy require the study of structure.

\section{Disclosure of Interest: None Declared}

\section{PL6 - GENETICS OF OSTEOPOROSIS: WHAT HAVE WE} LEARNT FROM GENOME-WIDE ASSOCIATION STUDIES? T. D. Spector ${ }^{1,} ;{ }^{1}$ Twin Research and Genetic Epidemiology, King's College London, London, United Kingdom

\footnotetext{
Abstract: Osteoporosis is a common heritable trait. In the last 2 years several large multicentre Genome-wide association studies (GWAS) for BMD and fractures were published using 300600,000 SNP gene markers in each subject. The first from an Anglo-Dutch group used Twins as the discovery sample and a total of 8500 subjects. Two strong signals emerged which reached genome-wide significance - one from the LRP5 gene and the other Osteoprotegerin - a combination of both risk allelles being present in 1 in 4 women and conferring a risk of 1.5 -fold risk of fracture. The other study published at the same time used 11,000 Icelandic, and European samples also showed the importance of the OPG/RANKL system, as well as the Estrogen receptor ESR1 and two new loci. Other smaller GWAS have found a few extra gene loci. A meta-analysis GWAS including most of the groups involved in the world of over 40,000 subjects (GEFOS) has confirmed over 20 new loci for BMD and fracture. Studies of Asian populations have generally replicated most of the European loci and found a few ethnic specific loci. Similar successful collaborative GWAS have found loci in related traits - such as menopausal age, smoking, body fat, hip geometry, vitamin D levels etc.

These results have shown that first large numbers and collaborations are needed to produce replicable results and that these scans have identified the major pathways in bone metabolism - suggesting that any novel loci picked up may also have therapeutic potential.

Another meta-analysis from the same GEFOS group of 20,000 subjects looked at 150 candidate genes for bone density- and found only 9 which were significantly associated. Amongst the negative findings was the VDR gene- despite hundreds of previous papers. This suggests that most of the previous candidate gene literature is flawed because of publication bias and that the effect sizes of individual genes were usually too small to be detected. With over 25 common gene loci now identified - the search is to understand their role and the source of the large unexplained genetic variance - that still remains to be found.
}

1. Richards JB et al. Lancet 2008;371:1505

2. Styrskarsdottir U et al. NEJM 2008;358:2355

3. Rivadeneira F et al. Nat Genet. 2009;41:1199

4. Kung AW et al. Am J Hum Gen 2010 (in press)

5. Richards JB et al . Ann Int med 2009;151:528

Disclosure of Interest: None Declared

\section{PL7 - INTERACTION BETWEEN BONE AND JOINTS IN PHYSIOLOGY AND DISEASE}

G. Schett ${ }^{1, *} ;{ }^{1}$ University of Erlangen-Nuremberg, Erlangen, Germany

Abstract: Arthritis is a perfect example for the clinical relevance of the interaction between inflammation and bone. Immune activation and inflammation in the course of arthritis affect profoundly influence bone remodelling. Typically, patients with rheumatoid arthritis suffer from bone erosions resulting from an excess of bone resorption. In rheumatoid arthritis proinflammatory cytokines such as TNF, IL-1 and IL-17 as well as PGE2 promote the synthesis of RANKL in the synovial membrane, which allows the local differentiation of osteoclasts and damage to the adjacent cortical bone surface. Anti-osteoclastogenic cytokines such as IFN $\gamma$, IL-4 and IL-10 do not compensate the effect of these proinflammatory mediators. Moreover, proinflammatory cytokines, in particular TNF, enhance the expression of Dkk-1 and sclerostin, which act as Wnt antagonists and effectively block bone resorption. Thus, inflammation in the context of rheumatoid arthritis enhances bone resorption while blocking bone formation and thus prevents adequate repair responses. In contrast to rheumatoid arthritis, other forms of joint inflammation such as spondylarthritides are associated with excessive bone formation at periosteal sites close to inflamed joints and the enthesial organs. Clinically these lesions appear as bony spurs. These bony spurs are based on enhanced bone formation, which is driven by the expression of bone morphogenic proteins as well as Wnt proteins. In summary, bone remodelling is crucial for the morphological differences of the various forms of arthritis. Insights into the molecular regulation of bone remodelling in arthritis allows to define specific therapeutic interventions to protect joints form inflammatory damage.

\section{Disclosure of Interest: None Declared}

\section{PL8 - GLITAZONE AND BONE: A PARADIGM TO BETTER UNDERSTAND BONE PHYSIOLOGY}

C. J. Rosen ${ }^{1, *} ;{ }^{1}$ Maine Medical Center Research Institute, Scarborough, Maine, United States

Abstract: The discovery of the thiazolidinediones (TZDs) for the treatment of insulin resistance informed us about the important role of nuclear receptors in regulating cell metabolism. PPARG is both a nuclear receptor and transcriptional regulator of multiple processes related to both lipid storage and insulin sensitivity. The TZDs are potent ligands for PPARG, but other activators include endogenous substances such as the prostaglandins and 
fatty acids. Stimulation of PPARG in adipocytes leads to multiple downstream targets that stimulate lipid biosynthesis and storage. Adipocytes and osteoblasts arise from the same multipotent stem cell but then enter lineages based upon specific transcription factors, co-activators, and co-repressors. PPARG is one of those determinants of stem cell fate and treatment with the glitazones provide significant insights into the activation of PPARG albeit in a pharmacologic manner. Rosiglitazone, the prototypical TZD, dramatically improves insulin sensitivity but also increases weight gain as the dual function of PPARG (enhanced glucose tolerance and lipid storage)is fully activated. At the same time, there are significant changes in skeletal metabolism that in the long run are detrimental. In particular, marrow adipogenesis increases while bone formation is suppressed and bone resorption is activated. Understanding the downstream targets of PPARG activation in progenitor cells are critical in determining lineage allocation. Several of these will be discussed as potential targets to preserve bone mass and promote insulin sensitivity. The ideal glitazone would be one that utilized the insulin arm of the PPARG targets while not activating adipogenesis and preventing osteogenesis.

\section{Disclosure of Interest: None Declared}

\section{PL9 - EXTRASKELETAL EFFECTS OF VITAMIN D}

B. Dawson-Hughes ${ }^{1, *} ;{ }^{1}$ USDA Human Nutrition Research Center on Aging, Tufts University, Boston, United States

\begin{abstract}
In addition to its classical effects on bone and muscle, vitamin $\mathrm{D}$ has been associated with a number of chronic diseases, including diabetes, cancer, cardiovascular disease, and infection. Mechanisms may involve effects of vitamin D and its metabolites on inflammation and on cell differentiation, proliferation, and apoptosis. Diabetes currently affects 285 million people worldwide at a cost of $11.6 \%$ of the total world health expenditure. Observational studies show a fairly consistent association between low vitamin $\mathrm{D}$ status and both prevalent and incident type two diabetes mellitus (t2DM). Evidence from post hoc analyses of vitamin $\mathrm{D}$ and/or calcium supplementation trials suggests that combined vitamin $\mathrm{D}$ and calcium supplementation may have a role in the prevention of $\mathrm{t} 2 \mathrm{DM}$, particularly in high-risk populations with glucose intolerance. In 2010, cancer will become the world's leading cause of death. It has been estimated that one third of cancer deaths may be attributable to diet. Higher levels of 25OHD have been linked to lower rates of cancer of the colon, prostate, breast, and lung. For prostate and breast cancer, there appears to be a U-shaped association, with cancer mortality declining as $25 \mathrm{OHD}$ levels approach 60 to $100 \mathrm{nmol} / \mathrm{L}$ and increasing as they exceed this range. In contrast, serum $25 \mathrm{OHD}$ may be positively associated with pancreatic cancer incidence rates, at least in male smokers. Polymorphisms in the vitamin D receptor gene seem to affect cancer risk, particularly at low calcium intake levels. In the one available prospective calcium and vitamin $\mathrm{D}$ intervention trial, supplementation appeared to reduce the incidence of all cancers, but this finding needs to be confirmed in larger studies. Finally, vitamin D insufficiency has been associated with several autoimmune diseases and infections, and with hypertension and cardiovascular disease. Highlights of the clinical evidence will be
\end{abstract}

reviewed. In conclusion, evidence linking vitamin D insufficiency to chronic disease progression is based largely on association studies. These studies must be interpreted with caution because of their inability to account entirely for the impact of powerful known confounders. The true effect of vitamin D on chronic disease risk and progression will remain unknown until randomized, controlled clinical trials are performed.

\section{Disclosure of Interest: None Declared}

\section{PL10 - STIMULATORS/INHIBITORS OF BONE TURNOVER: TOGETHER OR ONE AFTER ANOTHER?}

J. P. Bilezikian ${ }^{1, *} ;{ }^{1}$ Medicine, Columbia University College of Physicians \& Surgeons, New York, United States

Abstract: The available drugs for the treatment of osteoporosis can be categorized as either inhibitors of bone turnover or stimulators of bone turnover. The former group constitutes most of the registered treatments (e.g., bisphosphonates) while in the latter group parathyroid hormone, either as the full length molecule $[\mathrm{PTH}(1-84)]$ or the foreshortened peptide $[\mathrm{PTH}(1-34)]$ are the best examples. Since the availability of these two forms of parathyroid hormone, used as osteoanabolic agents, it has been attractive to consider combination therapy with an antiresorptive in order to take advantage of mechanisms that are different but potentially complementary. However, using a simultaneous combination approach with alendronate and $\mathrm{PTH}(1-84)$, the results as demonstrated by DXA and cQCT were better with PTH(1-84) monotherapy alone. A less potent antiresorptive, such as raloxifene with teriparatide gave better results suggesting that an antiresorptive of lower potency (raloxifene instead of alendronate) could augment, and not diminish, the actions of PTH. Sequential approaches to combination therapy have employed antiresorptives prior to using an osteoanabolic. The data here suggest, similar to the simultaneous combination approaches, that the stronger the antiresorptive effect, the greater the likelihood for an attenuated effect of the osteoanabolic used subsequently. Variations on this theme of sequential combination therapy have used cyclic approaches in which a hiatus, without anabolic therapy, spans two distinct periods of anabolic therapy. In some protocols, the hiatus has been free of an antiresorptive while in other protocols the antiresorptive is present throughout. Still other studies have directly compared 'switching' from an antiresorptive to an osteoanabolic vs. 'adding' the osteoanabolic to the antiresorptive regimen. The results from these various studies are providing insights into conditions under which combination therapy might be more effective than monotherapy alone. Finally, the data are clear that when osteoanabolic therapy is used initially for treatment of osteoporosis, it must be followed by antiresorptive therapy in order to maintain the densitometric gains achieved with the use of the osteoanabolic alone. As other approaches to combination therapy are tested, we may yet realize a way of optimizing the use of these two classes of drugs for the treatment of osteoporosis.

Disclosure of Interest: Grant / Research Support from: NPS, Consultant / Speaker's bureau / Advisory activities with: Eli Lilly, Alliance for Better Bone Health, Merck, Novartis, Amgen, NPS 


\section{PL11 - DEVELOPMENTAL PLASTICITY AND LATER OSTEOPOROSIS}

M.A. Hanson ${ }^{1, *} ;{ }^{1}$ Developmental Origins of Health and Disease Division, University of Southampton, Southampton, United Kingdom

\begin{abstract}
Epidemiological and animal studies show that small changes in the environment during development, e.g. in nutrient provision or balance, induce phenotypic changes which affect an individual's responses to its later environment. These may in turn alter the risk of chronic disease resulting from inadequate responses, e.g. to a rich environment leading to metabolic syndrome or cardiovascular disease. Recent work demonstrates an important role for molecular epigenetic processes in producing such effects, processes which are targeted to promoter regions of specific genes in specific tissues but which also include changes in histone structure and post-transcriptional processes involving miRNAs. Such fine control of gene expression endorses the view that the mechanisms have been retained through evolution as a result of the adaptive advantage which they confer, rather than representing extreme effects of developmental disruption akin to teratogenesis. Population groups migrating to new environments can have increased risks of chronic disease. The effects on individual tissues and organs are part of a lifecourse strategy, through which reproduction is traded off against bone composition, growth and repair. Developmental plasticity can therefore have consequences for ageing and for the timing and severity of associated chronic disease. Body composition changes include those affecting fat, skeletal muscle and bone. Recent studies reveal developmental effects on later bone density and composition (both mineral content and fat deposition), muscle mass and fibre type and muscle capillary density. Risks of fracture and arthritic damage are amplified by obesity, which also has a developmental origin. New research shows that biomarkers, especially epigenetic changes, at birth can be powerful predictors of later risk. This opens a new avenue for targeted interventions to reduce such risks, to monitor their efficacy and to give mechanistic insights. MAH is supported by the British Heart Foundation
\end{abstract}

Disclosure of Interest: None Declared

\section{PL12 - EMERGING THERAPIES FOR OSTEOPOROSIS}

S.E. Papapoulos ${ }^{1, *} ;{ }^{1}$ Endocrinology \& Metabolic Diseases, Leiden University Medical Center, Leiden, Netherlands

Abstract: During the past few years there have been significant developments in the pharmacotherapy of osteoporosis. These developments were paralleled by significant progress in our understanding of the local regulation of bone metabolism. Particularly, studies of human and animal genetics have led to identification of novel, more specific, signalling pathways in bone cells that can provide targets for new therapeutics for osteoporosis.

Such novel targets in osteoclasts include, among others, RAN$\mathrm{KL}$ and cathepsin-K. A fully human monoclonal antibody to RANKL (denosumab) was developed and approved in Europe for the treatment of osteoporosis while cathepsin-K inhibitors have been evaluated in phase 2 studies and one of them (odanacatib) is currently in phase 3 clinical development.

The PTH paradigm illustrated the possibility of stimulating bone formation in osteoporotic patients and opened the way for the development of bone forming agents and novel forms of PTH or PTHrP are at different stages of clinical development. A particularly interesting approach has been the development of molecules that antagonize the calcium sensing receptor of the parathyroid cells and stimulate PTH secretion (calcilytics). The most exciting development of recent years has been, however, the recognition of the central role of the Wnt signalling pathway in bone formation which, in turn provided, a number of attractive targets for the development of pharmaceuticals. For example, inhibition of this pathway by blocking the action of sclerostin represents a very promising novel approach to stimulating bone formation in patients with osteoporosis.

These new developments may allow in the future tailoring pharmacotherapy to the specific needs and pathophysiological profile of the individual patient. However, apart from establishing the efficacy of these new molecules a critical issue for their introduction into clinical practice will be their tolerability and safety profile.

Disclosure of Interest: Grant / Research Support from: Procter \& Gamble Pharmaceuticals, Consultant / Speaker's bureau / Advisory activities with: Amgen, GSK, Merck \& Co, Novartis, P\&G, Roche, Wyeth, Member of: IOF, ECTS 
